\title{
Assessment of Zymomonas mobilis biotechnological potential in ethanol production by flux variability analysis
}

\author{
Agris Pentjuss ${ }^{1,2 *}$, Uldis Kalnenieks ${ }^{3}$ \\ ${ }^{1}$ Biosystems Group, Department of Computer Systems, Latvia University of Agriculture, Liela iela 2, LV3001, Jelgava, Latvia \\ ${ }^{2}$ SIA TIBIT, Dobeles iela 10-9, LV3001, Jelgava, Latvia \\ ${ }^{3}$ Institute of Microbiology and Biotechnology, Faculty of Biology, Latvia University, Kronvalda bulv. 4, LV-1586, Riga, Latvia \\ *Corresponding author \\ agris.pentjuss@gmail.com
}

Received: 23 May 2014; accepted: 30 May 2014; published online: 31 May 2014.

This paper has no supplementary material.

\begin{abstract}
Metabolic engineering of microorganisms is a promising direction in industrial biotechnology. Engineering approach requests finding the best possible alternative of metabolic pathway fluxes, with preset limitations for productivity, yield, set of byproducts, robustness and other features. In case of industrial applications the steady state precondition has to be taken into account, thus enabling application of stoichiometric modeling. Spectrum of solutions maximizing ethanol or succinate production from glucose or xylose is analyzed at fixed maximal production rate of products and for $80-100 \%$ range of maximal production rate to assess the robustness of yield to disturbances in the performance of reactions. Maximal production of desired components from glucose and xylose turned out to be very similar by the flux distribution with very limited ability to compensate throughput limitations of important reactions thus causing reduction of yield. Requesting the network performance at 80-100\% of yield leads to increase of robustness, and majority of reactions can be compensated by others to maintain the steady state at the desired yield level.
\end{abstract}

Keywords: Zymomonas mobilis, central metabolism, flux variability analysis, modeling, robustness.

\section{Introduction}

Ethanol and succinate production (Blank and Kuepfer, 2010; Dien et al., 2003; Hübner et al., 2011; Lee et al., 2010; Patil et al., 2004) by metabolically engineered microorganisms (Nielsen and Keasling, 2011; Pharkya and Maranas, 2006) is a perspective direction in biotechnology.

Often when optimizing flux distribution, only the best flux is assessed (Pentjuss, et al., 2013a). More advanced approach is to look for the best possible flux distribution, when biomass growth is taking place, to make the bioconversion more efficient. Another step forward is to ensure that even the worst possible flux distribution at growing biomass conditions give industrially feasible yield (Trinh et al., 2008). That can be done using elementary mode analysis (Trinh et al., 2009) or flux variability analysis (FVA) (Boele et al., 2012; Orth et al., 2010). Elementary mode analysis requests cumbersome calculations, while FVA can be performed with small computational effort (Boele et al., 2012; Gudmundsson and Thiele, 2010; Kostromins, 2012; Orth et al., 2010; Pentjuss, et al., 2013b), and gives good overview of the range of possibilities and stability of possible solutions. Particular solutions can be visualized at different scales (Kostromins and Stalidzans, 2012) and analysed more closely.

Recent progress with stoichiometric modeling of bacteria Zymomonas mobilis enables application of the FVA approach. The first stoichiometric models of Z. mobilis were published without available metabolic model files (Lee et al., 2010; Tsantili et al., 2007; Widiastuti et al., 2011) and therefore were not directly applicable for modeling and optimization. In this study the stoichiometric model of $Z$. mobilis central metabolism (Pentjuss, et al., 2013a) is used. The model does not include the fluxes from the central metabolism to biomass growth, and therefore would be valid for non-growing cells, e.g. for immobilized culture. So far for this model published optimization results (Pentjuss, et al., 2013a) show just one of the optimal solutions without analysis of the whole spectrum of possible flux distributions.

Still it has to be noted that stoichiometric modeling does not take into account thermodynamic features of enzymes and realistic production rate depends on the values of kinetic parameters (Kalnenieks et al., 2014). Therefore, the outcomes of stoichiometric modeling have to be treated as the yield values that definitely can not be exceeded, but could remain unreachable.

In this study we aim to apply a more detailed approach to the central metabolism model of Z. mobilis (Pentjuss, et al., 2013a) to discover the full spectrum of optimal solutions and their robustness in terms of alternatives in flux distributions for production of ethanol and succinate from glucose and xylose.

\section{Materials and methods}

\subsection{Model of Z.mobilis central metabolism and task setting}

Stoichiometric model of Z. mobilis central metabolism (Pentjuss, et al., 2013a) as published in supplementary materials (Pentjuss, et al., 2013a) is used for optimization of ethanol and succinate production from glucose and xylose. The model includes 58 reactions of central metabolic pathways and 21 transmembrane transport reactions. Additionally, 21 virtual 
exchange reactions are introduced according to the protocol of model development (Thiele and Palsson, 2010). For analysis purpose 4 reactions, present in the published reconstruction of the central metabolism (Pentjuss, et al., 2013a) were knocked out (Glycerate kinase (E.C. 2.7.1.31), heterologous 3phosphoglycerate phosphatase (E.C. 3.1.3.38), citrate lyase (E.C. 4.1.3.6) and 6-phosphogluconate dehydrogenase (E.C. 1.1.1.44)). Also, model was extended with additional reactions (glycerol-3-phosphate dehydrogenase [NADH+] E.C. 1.1.1.8 (G3PD2), glutamate dehydrogenase E.C. 1.4.1.3 (GD2), malate dehydrogenase (oxaloacetate-decarboxylating) NADP+ E.C. 1.1.1.40 (MEMDLD)) to cover the possible redoxbalancing reactions in $Z$. mobilis.

FVA was performed at substrate input flux of $100 \mu \mathrm{mol}$ $\mathrm{gDW}^{-1} \mathrm{hr}^{-1}$ at: 1) maximal value of product yields and 2) 80$100 \%$ of maximal value of desired product yields. Four substrate-product pairs were evaluated: glucose-ethanol, glucose-succinate, xylose-ethanol and xylose-succinate.

\subsection{Analysis methods and software tools}

ScrumPy software (Poolman, 2006) was used for modeling and analysis. Flux variability analysis (Burgard et al., 2001) method was implemented into ScrumPy. Visualization was carried out by Linux compatible graphic utility Gnuplot (http://www.gnuplot.info/) with Gnuplot.py (http://gnuplotpy.sourceforge.net/) library using CandleSticks option.

Alternatively, the experiment could be performed also by COBRA Toolbox 2.0 (Schellenberger et al., 2011).

\section{Results and discussion}

The classical flux variability analysis goal is to get an overview about the possible flux distributions at the maximal yield of the desired product (Fig. 1 and Fig. 2). The 54 central metabolic pathways reactions are placed on the left side of the horizontal axis of figures followed by 19 transmembrane transport reactions. The virtual exchange reactions are not introduced in the figures. The variability of reactions is summarized in Table 1.

\subsection{Flux variability at maximal production rate of products}

Maximal yield of products can be determined by optimizing production of a particular product and setting that value as a requested limitation during flux variability analysis.

\subsubsection{Production of ethanol}

Flux variability analysis at maximal production yield of ethanol demonstrates just seven reactions for substrates with rates that may vary in a relatively wide range. Thus, reduction of flux of one of those reactions may be compensated by one or more of the others. The difference between glucose and xylose as substrates is small as it can be seen in Fig. 1a and Fig. 1b.

There are 31 and 30 reactions without flux for glucose and xylose correspondingly. At the same time the number of variable reactions within $10 \mu \mathrm{mol} \mathrm{gDW}^{-1} \mathrm{hr}^{-1}$ are 10 and 3 for glucose and xylose, correspondingly. That indicates a higher robustness of ethanol production from glucose, than from xylose.

\subsubsection{Production of succinate}

Flux variability analysis at maximal production yield of succinate (Fig. 1c and Fig. 1d) indicates significant variability opportunities of 13 reactions for both substrates. There are 29 and 8 reactions without flux for glucose and xylose correspondingly indicating higher metabolic load and lower robustness in case of xylose. At the same time the number of variable reactions within $10 \mu \mathrm{mol} \mathrm{gDW}^{-1} \mathrm{hr}^{-1}$ are 2 and 14 for glucose and xylose, correspondingly, indicating higher robustness in case of xylose.

\subsection{Flux variability at $80-100 \%$ of maximal yield of products}

To see the robustness of the system under lower yield of products, the FVA was repeated, setting the upper/lower boundary limits of products to $80-100 \%$ of maximal yield. Regarding the byproducts spectrum, there are very wide options: acetate, acetoin, acetaldehyde, pyruvate, glutamate, succinate, dihydroacetone.

\subsubsection{Production of ethanol}

During production of ethanol at $80-100 \%$ of maximal yield (Fig. 2a and Fig. 2b) there are 35 reactions with significant variability for both substrates. Just 4 and 11 reactions have a fixed non-zero value for glucose and xylose as substrates, respectively, indicating the small level of robustness. There are 15 and 8 reactions with zero value for glucose and xylose, correspondingly, showing the number of reactions with no influence on product flux.

\subsubsection{Production of succinate}

The production of succinate at $80-100 \%$ level of maximal yield (Fig. 2c and Fig. 2d) also becomes much more robust, than at set maximal product yield, having acetate, acetoin, acetaldehyde, pyruvate, glutamate, succinate, dihydroacetone as the possible byproducts. The summary of variability of succinate production (Table 1) is identical to the one for ethanol for both substrates.

Variability of 54 central metabolic network reactions depending on substrate-product pair. Transmembrane transport reactions are not included.

\begin{tabular}{|c|c|c|c|c|c|c|}
\hline $\begin{array}{l}\text { Substrate-product } \\
\text { pair }\end{array}$ & $\begin{array}{l}\text { Yield (\% of } \\
\text { max })\end{array}$ & $\begin{array}{l}\text { Reactions with } \\
\text { variability } \\
\text { above } 10 \mu \mathrm{mol} \\
\mathrm{gDW}^{-1} \mathrm{hr}^{-1}\end{array}$ & $\begin{array}{l}\text { Reactions with varia- } \\
\text { bility below } 10 \mu \mathrm{mol} \\
\mathrm{gDW}^{-1} \mathrm{hr}^{-1}\end{array}$ & $\begin{array}{c}\text { Constant } \\
\text { nonzero flux }\end{array}$ & No flux & $\begin{array}{l}\text { Total reactions except } \\
\text { substrate and product }\end{array}$ \\
\hline Glucose-ethanol & 100 & 7 & 10 & 6 & 31 & 54 \\
\hline Xylose-ethanol & 100 & 7 & 3 & 14 & 30 & 54 \\
\hline Glucose-succinate & 100 & 13 & 2 & 10 & 29 & 54 \\
\hline Xylose-succinate & 100 & 13 & 14 & 19 & 8 & 54 \\
\hline Glucose-ethanol & $80-100$ & 35 & 0 & 4 & 15 & 54 \\
\hline Xylose-ethanol & $80-100$ & 35 & 0 & 11 & 8 & 54 \\
\hline Glucose-succinate & $80-100$ & 35 & 0 & 4 & 15 & 54 \\
\hline Xylose-succinate & $80-100$ & 35 & 0 & 11 & 8 & 54 \\
\hline
\end{tabular}


a
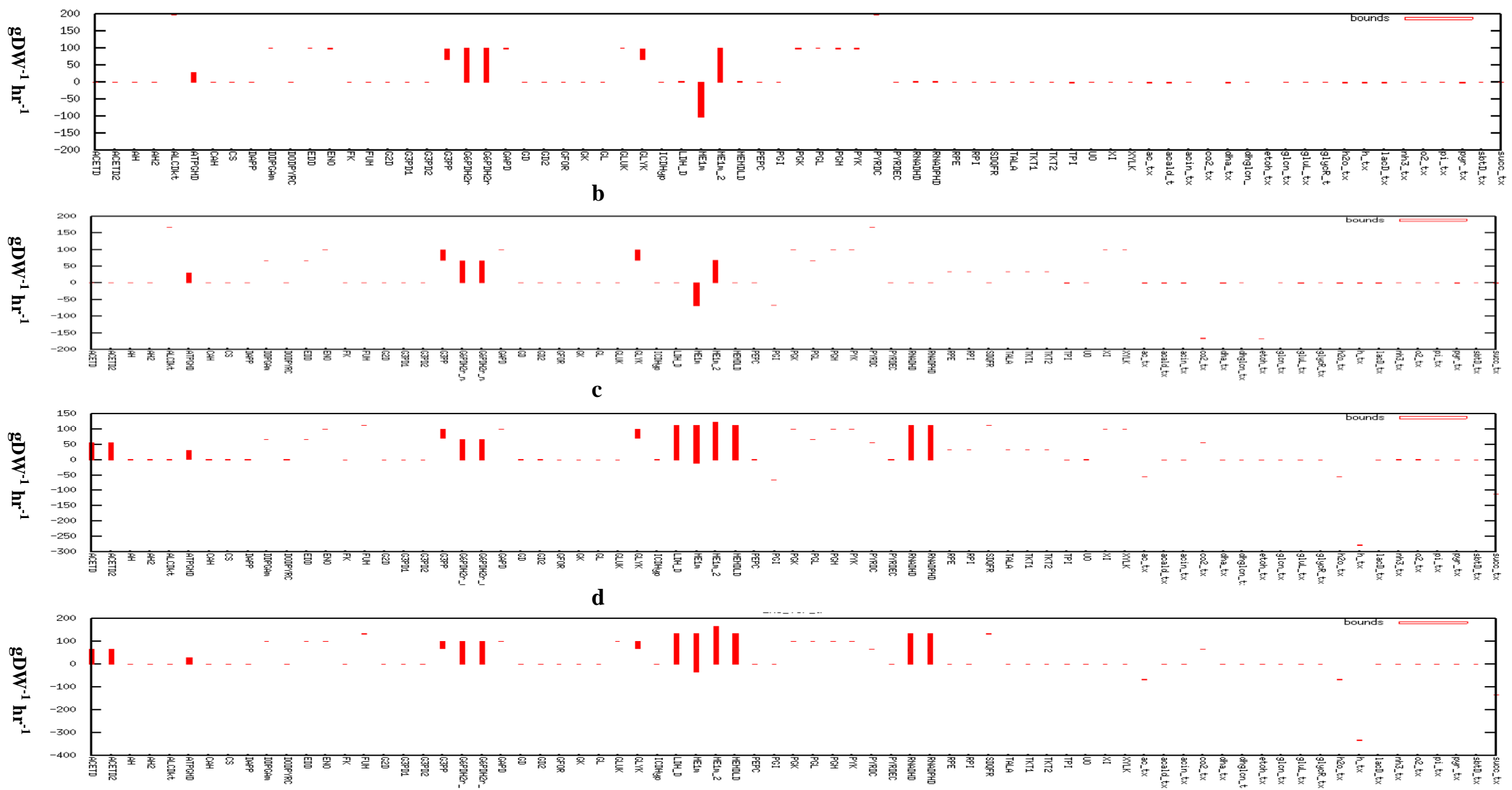

Fig. 1. Flux variability analysis for substrate-product pairs glucose-ethanol (a), xylose-ethanol (b), glucose-succinate (c), xylose-succinate (d) at maximal product production and fixed substrate consumption rate $100 \mu \mathrm{mol} \mathrm{gDW}^{-1} \mathrm{hr}^{-1}$. 


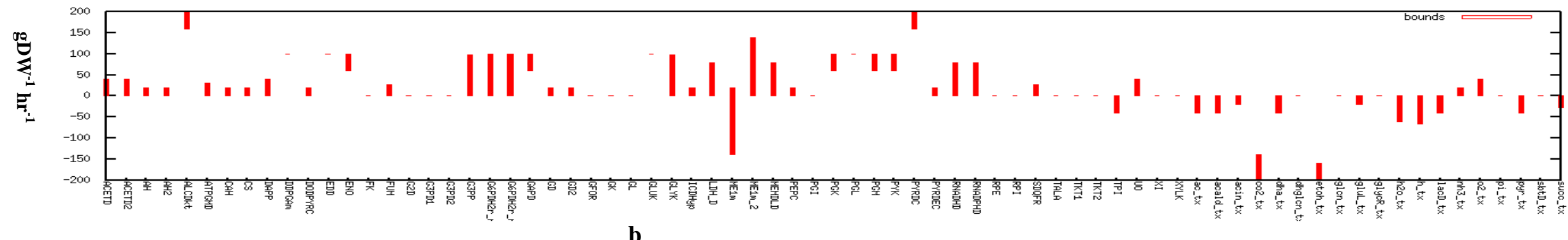

b
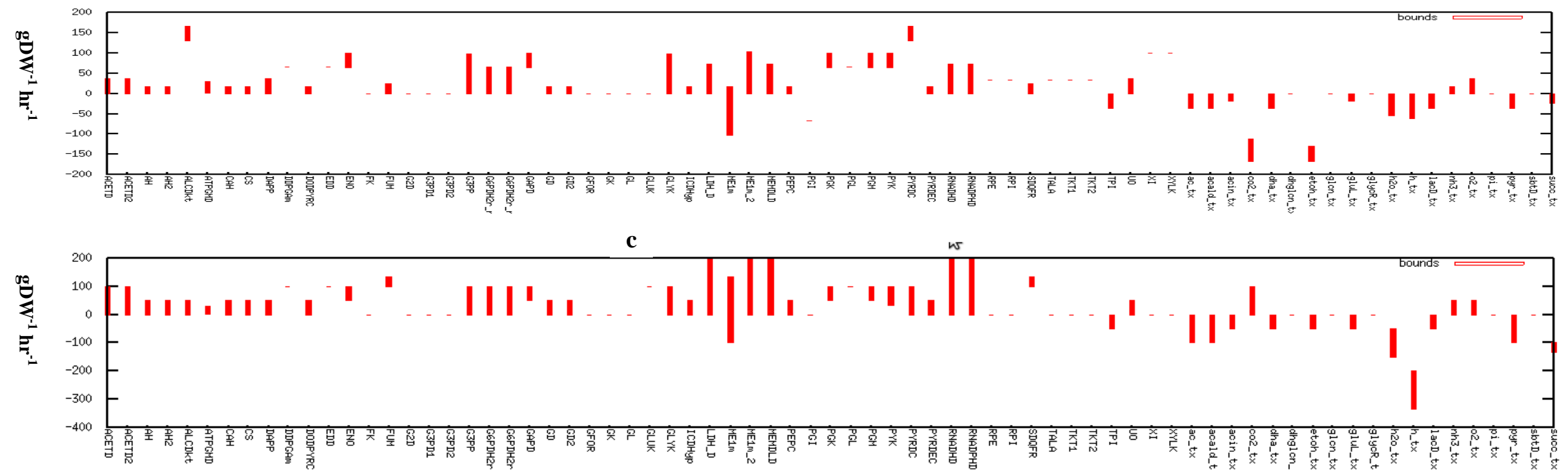

d

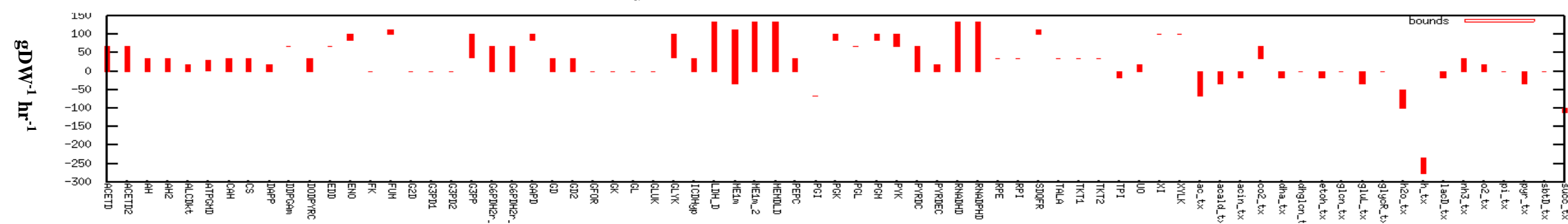

Fig.2. Flux variability analysis for substrate-product pairs glucose-ethanol (a), xylose-ethanol (b), glucose-succinate (c), xylose-succinate (d) at 80-100\% of maximal product production and fixed substrate consumption rate $100 \mu \mathrm{mol}_{\mathrm{gDW}} \mathrm{hr}^{-1}$ 


\subsubsection{Comparative robustness of substrate-product pairs}

As expected the robustness or tolerance to failures or flux reductions of different reactions increase if the requested product yield range is widened. Among the $100 \%$ yield substrate-product pairs the most robust seems to be the combination is xylose-succinate pair with 27 variable reactions. At the same time the low number of no flux reactions decreases the robustness of yield.

Widening the requested yield range to $80-100 \%$ from maximal the number of variable reactions increases up to 35 for all four combinations and there are no reactions with variability below $10 \mu \mathrm{mol} \mathrm{gDW}^{-1} \mathrm{hr}^{-1}$. Glucose as a substrate give more robustness having 15 zero flux reactions in contrast to xylose-based processes with 8 zero flux reactions.

\section{Conclusion}

Concentration on analysis of maximal yield of the desired product excludes the issue of robustness from analysis, although it is very important in industrial environment. Flux variability analysis (FVA) is one of the tools to assess the robustness and fragility of the metabolic process of interest. Main indicators for robustness are the number of reactions varying in wide range and the number of zero flux reactions, while the number of constant nonzero flux and reactions with low variability indicate fragility of the process of interest.

At maximal yield of products there often are very limited alternatives for the main production pathway. Reaching maximal product yield both in case of ethanol and succinate production Z. mobilis metabolic network shows small redundancy in network flux distribution, but lowering the requested ethanol or succinate production yield, the redundancy in byproduct fluxes increases. The number of reactions with variability above $10 \mu \mathrm{mol} \mathrm{gDW}^{-1} \mathrm{hr}^{-1}$ increases from 7 and 13 for ethanol and succinate production to 35 widening the requested range of yield from $100 \%$ to $80-100 \%$.

Reduction of the requested product yield and varying the set of byproducts in the model might be a reasonable approach to keep the economical benefits at acceptable level and increase the redundancy of the microbial producer strain.

\section{References}

Blank, L.M. and Kuepfer, L. (2010), "Metabolic flux distributions: genetic information, computational predictions, and experimental validation.", Applied microbiology and biotechnology, Vol. 86 No. 5, pp. 1243-55. http://dx.doi.org/10.1007/s00253-010-2506-6

Boele, J., Olivier, B.G. and Teusink, B. (2012), "FAME, the Flux Analysis and Modeling Environment.", BMC systems biology, Vol. 6 No. 1, p. 8. http://dx.doi.org/10.1186/1752-0509-6-8

Burgard, A.P., Vaidyaraman, S. and Maranas, C.D. (2001), "Minimal reaction sets for Escherichia coli metabolism under different growth requirements and uptake environments.", Biotechnology progress, Vol. 17 No. 5, pp. 791-7. http://dx.doi.org/10.1021/bp0100880

Dien, B.S., Cotta, M. a and Jeffries, T.W. (2003), "Bacteria engineered for fuel ethanol production: current status.", Applied microbiology and biotechnology, Vol. 63 No. 3 , pp. 258-66. http://dx.doi.org/10.1007/s00253-003-1444-y

Gudmundsson, S. and Thiele, I. (2010), "Computationally efficient flux variability analysis.", BMC bioinformatics, BioMed Central Ltd, Vol. 11 No. 1, p. 489. http://dx.doi.org/10.1186/1471-2105-11-489

Hübner, K., Sahle, S. and Kummer, U. (2011), "Applications and trends in systems biology in biochemistry.", The FEBS journal, Vol. 278 No. 16, pp. 2767-857. http://dx.doi.org/10.1111/j.1742-4658.2011.08217.x

Kalnenieks, U., Pentjuss, A., Rutkis, R., Stalidzans, E. and Fell, D.A. (2014), "Modeling of Zymomonas mobilis central metabolism for novel metabolic engineering strategies", Frontiers in Microbiology, Vol. 5, http://dx.doi.org/10.3389/fmicb.2014.00042.

Kostromins, A. (2012), "Altfluxes: COBRA Toolbox extension for flux variability analysis of stoichiometric models of metabolism", 5th
International Scientific Conference on Applied Information and Communication Technologies, pp. 294-299.

Kostromins, A. and Stalidzans, E. (2012), "Paint4Net: COBRA Toolbox extension for visualization of stoichiometric models of metabolism.", $\begin{array}{lllllll}\text { Biosystems, } & \text { Vol. } & 109 & \text { No. } & 2, & \text { pp. } & 233-9\end{array}$ http://dx.doi.org/10.1016/j.biosystems.2012.03.002

Lee, K.Y., Park, J.M., Kim, T.Y., Yun, H. and Lee, S.Y. (2010), "The genome-scale metabolic network analysis of Zymomonas mobilis ZM4 explains physiological features and suggests ethanol and succinic acid production strategies.", Microbial cell factories, BioMed Central Ltd, Vol. 9 No. 1, p. 94. http://dx.doi.org/10.1186/1475-2859-9-94

Nielsen, J. and Keasling, J.D. (2011), "Synergies between synthetic biology and metabolic engineering.", Nature biotechnology, Vol. 29 No. 8, pp. 693-5. http://dx.doi.org/10.1038/nbt.1937

Orth, J.D., Thiele, I. and Palsson, B.O. (2010), "What is flux balance analysis?", Nature biotechnology, Vol. 28 No. 3, pp. 245-8. http://dx.doi.org/10.1038/nbt.1614

Patil, K.R., Akesson, M. and Nielsen, J. (2004), "Use of genome-scale microbial models for metabolic engineering.", Current opinion in $\begin{array}{lllllll}\text { biotechnology, } & \text { Vol. } & 15 & \text { No. } & 1, & \text { pp. } & 64-9 .\end{array}$ http://dx.doi.org/10.1016/j.copbio.2003.11.003

Pentjuss, A., Odzina, I., Kostromins, A., Fell, D.A., Stalidzans, E. and Kalnenieks, U. (2013a), "Biotechnological potential of respiring Zymomonas mobilis: a stoichiometric analysis of its central metabolism", Journal of Biotechnology, Vol. 165 No. 1, pp. 1-10. http://dx.doi.org/10.1016/j.jbiotec.2013.02.014

Pentjuss, A., Rubenis, O., Bauze, D., Aprupe, L. and Lace, B. (2013b), "Flux variability analysis approach of autism related metabolism in stoichiometric model of mitochondria", Biosystems and Information

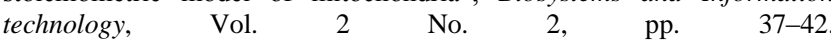
http://dx.doi.org/10.11592/bit.131102

Pharkya, P. and Maranas, C.D. (2006), "An optimization framework for identifying reaction activation/inhibition or elimination candidates for overproduction in microbial systems.", Metabolic engineering, Vol. 8 No. 1, pp. 1-13. http://dx.doi.org/10.1016/j.ymben.2005.08.003

Poolman, M.G. (2006), "ScrumPy: metabolic modelling with Python", IEE Proceedings - Systems Biology, Vol. 153 No. 5, p. 375.

Schellenberger, J., Que, R., Fleming, R.M.T., Thiele, I., Orth, J.D., Feist, A.M., Zielinski, D.C., et al. (2011), "Quantitative prediction of cellular metabolism with constraint-based models: the COBRA Toolbox v2.0", Nature Protocols, $\quad$ Vol. 6 No. 9, pp. 1290-1307. http://dx.doi.org/10.1038/nprot.2011.308

Thiele, I. and Palsson, B.O. (2010), "A protocol for generating a high-quality genome-scale metabolic reconstruction.", Nature protocols, Vol. 5 No. 1 , pp. 93-121. http://dx.doi.org/10.1038/nprot.2009.203

Trinh, C.T., Unrean, P. and Srienc, F. (2008), "Minimal Escherichia coli cell for the most efficient production of ethanol from hexoses and pentoses.", Applied and environmental microbiology, Vol. 74 No. 12, pp. 3634-43. http://dx.doi.org/ 10.1128/AEM.02708-07

Trinh, C.T., Wlaschin, A. and Srienc, F. (2009), "Elementary mode analysis: a useful metabolic pathway analysis tool for characterizing cellular metabolism.", Applied microbiology and biotechnology, Springer, Vol. 81 No. 5, pp. 813-826. http://dx.doi.org/10.1007/s00253-008-1770-1.

Tsantili, I.C., Karim, M.N. and Klapa, M.I. (2007), "Quantifying the metabolic capabilities of engineered Zymomonas mobilis using linear programming analysis.", Microbial cell factories, Vol. 6, $\quad$ p. 8 . http://dx.doi.org/10.1186/1475-2859-6-8

Widiastuti, H., Kim, J.Y., Selvarasu, S., Karimi, I. a, Kim, H., Seo, J.-S. and Lee, D.-Y. (2011), "Genome-scale modeling and in silico analysis of ethanologenic bacteria Zymomonas mobilis.", Biotechnology and bioengineering, Vol. 108 No. 3, pp. 655-65. http://dx.doi.org/10.1002/bit.22965 